NSF-ITP-97-110

\title{
Testing M(atrix) Theory at Two Loops
}

\author{
Katrin Becker* \\ Institute for Theoretical Physics \\ University of California \\ Santa Barbara, CA 93106
}

I discuss the relation between M-theory and M(atrix)-theory in flat space by considering the effective potential for the scattering of two groups of D0-branes in both theories. An explicit calculation of this potential up to two loop order in $\mathrm{M}$ (atrix)-theory reveals a fascinating agreement.

Lecture given at Strings '97; June 17, 1997.

\section{Introduction}

This lecture is based on two papers; a first paper that was written with Melanie Becker [1] and a second paper that we wrote with our collaborators Joe Polchinski and Arkady Tseytlin [2].

M-theory is our most promising candidate of being a quantum theory in eleven dimensions that includes gravity. Although we do not have a complete answer to the question what M-theory is we understand several aspects of it:

1. All different kinds of string theories can be obtained as different compactifications of M-theory [3].

2. At low energies and large distances Mtheory reduces to eleven-dimensional supergravity.

An important step towards understanding what M-theory is was made last year by Banks, Fischler, Shenker and Susskind 任. These authors conjectured that M-theory in the infinite momentum frame is described by a supersymmetric matrix model. The only dynamical degrees of freedom or partons are the D0-branes of Polchinski [5], so that the calculation of any physical quantity in M-theory can be reduced to a calculation in the $\mathrm{M}$ (atrix)-model quantum mechanics. The action describing a system of $N$ D0-branes can be regarded as ten-dimensional super Yang-Mills

*Address after Sep. 1, 1997: California Institute of Technology, Pasadena, CA 91125, USA theory dimensionally reduced to $0+1$ dimensions [6 9]. The bosonic part of this Lagrangian is

$$
\mathcal{L}=\frac{1}{2 R} \operatorname{Tr}\left(-\left(D_{\tau} X^{i}\right)^{2}+\frac{1}{2}\left[X^{i}, X^{j}\right]^{2}\right),
$$

where $R$ is the radius of the eleventh dimension; the signs are appropriate for antihermitian $X$ and $i=1, \ldots, 9$ denotes the transverse coordinates.

This quantum mechanical system has a $U(N)$ symmetry. While in the original formulation of the conjecture, which relates M-theory to $\mathrm{M}$ (atrix) theory, the $N \rightarrow \infty$ limit was implicit, a more recent formulation of the conjecture, due to Susskind [10,11], is valid for finite $N$. Susskind's new conjecture says: "The discrete light cone quantization of M-theory 12] is exactly described by the $U(N) \mathrm{M}$ (atrix) theory". The discrete light cone quantization agrees with the infinite momentum frame in the limit $N \rightarrow \infty$.

Our goal is to test this conjecture by making a precise comparison between M-theory and $\mathrm{M}$ (atrix)-theory for finite $N$. We will do so by computing the effective action for the scattering of two (groups of) D0-branes. We will see that the correspondence between both theories is correct even at two loops!.

Let me give an overview of my talk:

1. I will present the form of the super YangMills action in $0+1$ dimensions that governs the D0-brane behavior.

2. Derivation of the Feynman rules. 
3. Calculation of the one-loop effective action of $\mathrm{M}$ (atrix)-theory.

4. Calculation of the two-loop effective action of $\mathrm{M}$ (atrix)-theory.

5. I will show how the comparison with Mtheory works precisely and we will see how on the M-theory side we can make more predictions that will agree with $\mathrm{M}$ (atrix)theory calculations.

\section{Conclusions and Outlook.}

\section{Super Yang-Mills Action in 0+1 Dimen- sions}

To compute the effective action for two D0branes it will be convenient to work with the background field method 13,15 . This is a technique which allows us to fix a gauge and therefore do quantum computations without loosing explicit gauge invariance. The gauge theory action we are interested in can be obtained staring with ten-dimensional super Yang-Mills theory dimensionally reduced to $0+1$ dimensions. After gauge fixing the Lagrangian is

$\mathcal{L}=\operatorname{Tr}\left(\frac{1}{2 g} F_{\mu \nu}^{2}-i \bar{\psi} D \psi+\frac{1}{g}\left(\bar{D}^{\mu} A_{\mu}\right)^{2}\right)+\mathcal{L}_{\mathcal{G}}$

where $F_{\mu \nu}$ is a $U(2)$ field strength with $\mu, \nu=$ $0, \ldots, 9, \psi$ is a real sixteen component spinor and $\mathcal{L}_{\mathcal{G}}$ is the ghost lagrangian. For the gauge fixing term we will be using the background field gauge condition

$\bar{D}^{\mu} A_{\mu}=\partial^{\mu} A_{\mu}+\left[B^{\mu}, A_{\mu}\right]$,

where $B_{\mu}$ is the background field.

In $0+1$ dimensions we will use the following expressions for the field strength and the derivative of the fermionic field

$$
\begin{aligned}
F_{0 i} & =\partial_{\tau} X_{i}+\left[A, X_{i}\right], \\
F_{i j} & =\left[X_{i}, X_{j}\right], \\
D_{\tau} \psi & =\partial_{\tau} \psi+[A, \psi], \\
D_{i} \psi & =\left[X_{i}, \psi\right] .
\end{aligned}
$$

Here $A$ denotes the zero component of the gauge field appearing in (2). Setting $g=2 R$ we recover (1).
We would like to expand the action around a classical background

$X^{i}=B^{i}+\sqrt{g} Y^{i}$,

that describes the motion of two D0-branes on straight lines, where

$B^{1}=i \frac{v \tau}{2} \sigma^{3} \quad$ and $\quad B^{2}=i \frac{b}{2} \sigma^{3}$.

Here $v$ is the relative velocity of the two D0branes, $b$ is the impact parameter and $\sigma^{3}$ is a Pauli matrix. Furthermore $B^{i}=0$ for $i=0$ and $i=3, \ldots 9$. A convenient form of writing the action is in terms of $U(2)$ generators by decomposing the fields as 9]

$A=\frac{i}{2}\left(A_{0} \mathbb{1}+A_{a} \sigma^{a}\right)$,

and similarly for the fields $X^{i}$ and $\psi$. The zero components of this decomposition describe the motion of the center of mass and will be ignored in the following. The Lagrangian is now a sum of four terms

$\mathcal{L}=\mathcal{L}_{Y}+\mathcal{L}_{A}+\mathcal{L}_{\mathcal{G}}+\mathcal{L}_{\text {fermi }}$,

whose explicit form can be found in [1]. Here I only would like to mention that the bosonic lagrangians $\mathcal{L}_{Y}$ and $\mathcal{L}_{A}$ are described in terms of sixteen bosons with mass $m_{\mathcal{B}}^{2}=r^{2}=b^{2}+(v \tau)^{2}$, two bosons with $m_{\mathcal{B}}^{2}=r^{2}+2 v$, two bosons with $m_{\mathcal{B}}^{2}=r^{2}-2 v$ and ten massless bosons. All these fields are real. The ghost action is described in terms of two complex bosons with mass $m_{\mathcal{G}}^{2}=r^{2}$ and one complex massless boson.

\section{Feynman Rules}

There are two possible approaches to compute the gauge invariant background field effective action. The first one treats the background field exactly, so that this field enters in the propagators and vertices of the theory. To compute the effective action one has to sum over all 1PI graphs without external lines. The second approach treats the background field perturbatively, so that it appears as external lines in the 1PI graphs of the theory. We are following the first 
approach in which we treat the background field exactly.

We can now proceed to derive the Feynman rules. The explicit form of the vertices can be read off from the actions described a moment ago [1. The concrete form of the propagators can be easily obtained once we realize that a relation to the one-dimensional harmonic oscillator can be found. The propagators of all the bosonic fields take then the form

$$
\begin{aligned}
& \Delta_{\mathcal{B}}\left(\tau, \tau^{\prime} \mid \mu^{2}+(v \tau)^{2}\right)= \\
& \int_{0}^{\infty} d s e^{-\mu^{2} s} \sqrt{\frac{v}{2 \pi \sinh 2 s v}} \\
& \exp \left(-\frac{v}{2}\left(\frac{\left.\left(\tau^{2}+\tau^{\prime 2}\right) \cosh 2 s v-2 \tau \tau^{\prime}\right)}{\sinh 2 s v}\right)\right)
\end{aligned}
$$

where $\mu^{2}=b^{2}, b^{2} \pm 2 v$ depending on the type of boson that one is considering. The propagator of the fermionic fields is the solution to the equation

$$
\left(-\partial_{\tau}+m_{\mathcal{F}}\right) \Delta_{\mathcal{F}}\left(\tau, \tau^{\prime} \mid m_{\mathcal{F}}\right)=\delta\left(\tau-\tau^{\prime}\right),
$$

where $m_{\mathcal{F}}=v \tau \gamma_{1}+b \gamma_{2}$ is the fermionic mass matrix. Using the gamma matrix algebra it is easy to see that the fermionic propagator can be expressed through the bosonic propagator

$\Delta_{\mathcal{F}}\left(m_{\mathcal{F}}\right)=\left(\partial_{\tau}+m_{\mathcal{F}}\right) \Delta_{\mathcal{B}}\left(r^{2}-v \gamma_{1}\right)$.

This is a Dirac-like operator acting on a bosonic propagator of a particle with mass $r^{2}-v \gamma_{1}$. Since we have a closed expression for $\Delta_{\mathcal{B}}$, we therefore have a closed expression for $\Delta_{\mathcal{F}}$. Diagonalizing the mass matrix we find that our theory contains eight real fermions with mass $m_{\mathcal{F}}^{2}=r^{2}+v$ and eight real fermions with $m_{\mathcal{F}}^{2}=r^{2}-v$. The third component of $\psi$ is massless. With this Feynman rules we can now proceed to derive the effective actions. We will start with the one-loop effective action.

\section{One-Loop Effective Action}

In order to compute the one-loop effective action we are interested in the phase shift $\delta$ of one graviton scattered off a second one 13, 14,

$\delta=-\int d \tau V\left(b^{2}+v^{2} \tau^{2}\right)$.
The phase shift can be obtained from the determinants of the operators $-\partial_{\tau}^{2}+M^{2}$ that originate from integrating out the massive degrees of freedom at one-loop. The result for the one-loop determinants is 13

$$
\begin{aligned}
& \operatorname{det}^{4}\left(-\partial_{\tau}^{2}+r^{2}+v\right) \operatorname{det}^{4}\left(-\partial_{\tau}^{2}+r^{2}-v\right) \\
& \operatorname{det}^{-1}\left(-\partial_{\tau}^{2}+r^{2}+2 v\right) \operatorname{det}^{-1}\left(-\partial_{\tau}^{2}+r^{2}-2 v\right) \\
& \operatorname{det}^{-6}\left(-\partial_{\tau}^{2}+r^{2}\right) .
\end{aligned}
$$

In a proper time representation of the determinants the phase shift can be written as

$\delta=\int_{0}^{\infty} \frac{d s}{s} \frac{e^{-s b^{2}}}{\sinh s v}(3-4 \cosh s v+\cosh 2 s v)(1$

For large impact parameter the integrand can be expanded and one obtains for the leading order of the potential the result [4, 16]

$V(r)=\frac{15}{16} \frac{v^{4}}{r^{7}}$.

As argued by Banks, Fischler, Shenker and Susskind [4 this is precisely the result expected for a single supergraviton exchange in eleven dimensions. Therefore, the $(0+1)$-dimensional M(atrix)-model seems to know about the propagation of massless modes in eleven dimensions.

Next we would like to check if the agreement between both theories is specific to one-loop order or if it holds beyond that. We will do so by computing explicitly the two-loop effective action.

\section{Two-Loop Effective Action}

The two-loop effective action is given by the sum of all diagrams of the form contained in Figure 1.
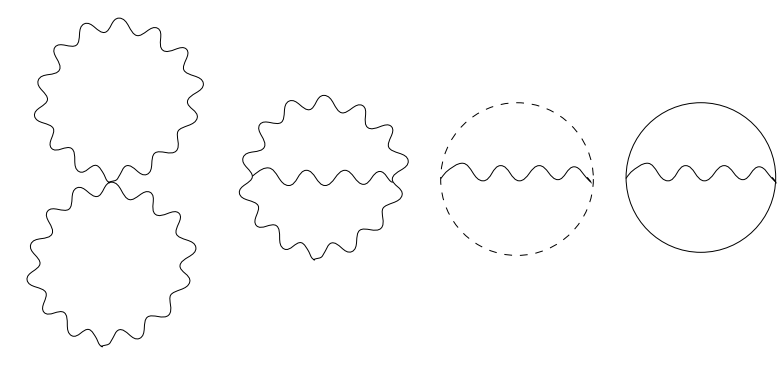

(a)

(b)

(c)

(d)

Figure 1 
The propagators for the fluctuations $Y$ and the gauge field $A$ are indicated by wavy lines, ghost propagators by dashed lines and the solid lines indicate the fermion propagators The explicit expressions for these graphs are

$\int d \tau \lambda_{4} \Delta_{1}\left(\tau, \tau \mid m_{1}\right) \Delta_{2}\left(\tau, \tau \mid m_{2}\right)$,

for the diagram involving the quartic vertex $\lambda_{4}$, where $\Delta_{1}$ and $\Delta_{2}$ are the propagators of the corresponding particles with masses $m_{1}$ and $m_{2}$ respectively and

$$
\begin{array}{r}
\int d \tau d \tau^{\prime} \lambda_{3}^{(1)} \lambda_{3}^{(2)} \Delta_{1}\left(\tau, \tau^{\prime} \mid m_{1}\right) \Delta_{2}\left(\tau, \tau^{\prime} \mid m_{2}\right) \\
\Delta_{3}\left(\tau, \tau^{\prime} \mid m_{3}\right)
\end{array}
$$

for the diagram involving the cubic vertices $\lambda_{3}^{(1)}$ and $\lambda_{3}^{(2)}$.

Let us see what kind of masses are involved in these diagrams. In equation (16) $m_{1}$ and $m_{2}$ are both different from zero. If, for example, $m_{1}$ is equal to zero we would have a massless state running in a loop, that gives a contribution

$\int \frac{d p}{p^{2}}$

to the relevant integrals. However, this expression vanishes in dimensional regularization. This is because of the following reason. The dimensional regularization of ill defined integrals is defined by three properties 22]:

1. translation,

2. dilatation,

3. factorization.

It is the invariance under dilatations that imposes the condition that the integral (18) vanishes. Therefore, diagrams containing a quartic vertex involve two massive particles. A similar argument for the diagram (17) leads to the conclusion that exactly one massless state is present.

From dimensional analysis we expect the twoloop effective action to be a series of the form

$\Gamma^{(2)}=g\left(\alpha_{0} \frac{1}{r^{2}}+\alpha_{2} \frac{v^{2}}{r^{6}}+\alpha_{4} \frac{v^{4}}{r^{10}}+\ldots\right)$.
Odd powers in $v$ in this series are missing because of time reversal invariance. The $\alpha_{i}$ 's are numerical coefficients that have to be determined from the computation of the Feynman diagrams. The details about the different contributions can be found in [1]. Here I only would like to mention the final results. First, the coefficient of the $v^{4} / r^{10_{-}}$ term appearing at two loops in matrix theory turns out to be equal to zero once all the contributions coming from bosons and fermions are added up. The vanishing of this numerical coefficient is in agreement with the non-renormalization theorem that was conjectured by Banks, Fischler, Shenker and Susskind [4] and it is required in order to have agreement with M-theory.

At this moment $\mathrm{M}$ (atrix)-theory has passed our first two-loop test: the vanishing of the $v^{4} / r^{10}$ term. However, this is only one term in the effective action of two D0-branes. By dimensional analysis we observe that the terms allowed in the double expansion in $v$ and $r$ take the following form

$$
\begin{aligned}
& \mathcal{L}_{0}=c_{00} v^{2} \\
& \mathcal{L}_{1}=c_{11} \frac{v^{4}}{r^{7}}+c_{12} \frac{v^{6}}{r^{11}}+c_{13} \frac{v^{8}}{r^{15}}+\ldots \\
& \mathcal{L}_{2}=c_{21} \frac{v^{4}}{r^{10}}+c_{22} \frac{v^{6}}{r^{14}}+c_{23} \frac{v^{8}}{r^{18}}+\ldots \\
& \mathcal{L}_{3}=c_{31} \frac{v^{4}}{r^{13}}+c_{32} \frac{v^{6}}{r^{17}}+c_{33} \frac{v^{8}}{r^{21}}+\ldots
\end{aligned}
$$

We have just seen that $c_{12}$ vanishes. Let's see how this result appears from the M-theory point of view. New surprises for other coefficients in (20) will appear!

\section{Comparison with M-theory and More Predictions}

We consider the scattering of two gravitons with momenta $p_{-}=N_{1} / R$ and $p_{-}=N_{2} / R$. We take $N_{1}$ to be large so that the first graviton is the source of the gravitational field. The source graviton is taken to have vanishing transverse velocity. Its worldline is $x^{-}=x^{i}=0$ and it produces the Aichelburg-Sexl metric 17.

$G_{\mu \nu}=\eta_{\mu \nu}+h_{\mu \nu}$, 
where the only nonvanishing component of $h_{\mu \nu}$ is

$h_{--}=\frac{2 \kappa_{11}^{2} p_{-}}{7 \omega_{8} r^{7}} \delta\left(x^{-}\right)=\frac{15 \pi N_{1}}{R M^{9} r^{7}} \delta\left(x^{-}\right)$.

Here $\kappa_{11}^{2}=16 \pi^{5} / M^{9}$ (see ref. [16] for example), $\mathrm{M}$ is the eleven-dimensional Planck mass up to a convention-dependent numerical factor and $\omega_{8}$ is the volume of the eight-sphere. This metric can be thought of as obtained from the Schwarzchild metric by taking the limit of infinite boost in the + direction while the mass is taken to zero; the latter accounts for the absence of higher-order terms in $1 / r$ or $N_{1}$. The source graviton is in a state of definite $p_{-}$and so we average over the $x^{-} \in(0,2 \pi R)$ direction to give

$h_{--}=\frac{15 N_{1}}{2 R^{2} M^{9} r^{7}}$.

For the action of the 'probe' graviton in this field we use the following trick. Begin with the action for a massive scalar (spin effects fall off more rapidly with $r$ ) in eleven dimensions

$$
\begin{aligned}
S & =-m \int d \tau\left(-G_{\mu \nu} \dot{x}^{\mu} \dot{x}^{\nu}\right)^{1 / 2} \\
& =-m \int d \tau\left(-2 \dot{x}^{-}-v^{2}-h_{--} \dot{x}^{-} \dot{x}^{-}\right)^{1 / 2}
\end{aligned}
$$

where we have used the form of the AichelburgSexl metric. A dot denotes $\partial_{\tau}$ and $v^{2}=\dot{x}^{i} \dot{x}^{i}$. This action vanishes if we take $m \rightarrow 0$ with fixed velocities, but for the process being considered here it is $p_{-}$that is to be fixed. We therefore carry out a Legendre transformation on $x^{-}$:

$p_{-}=m \frac{1+h_{--} \dot{x}^{-}}{\left(-2 \dot{x}^{-}-v^{2}-h_{--} \dot{x}^{-} \dot{x}^{-}\right)^{1 / 2}}$.

The appropriate Lagrangian for $x^{i}$ at fixed $p_{-}$is (minus) the Routhian,

$\mathcal{L}^{\prime}\left(p_{-}\right)=-\mathcal{R}\left(p_{-}\right)=\mathcal{L}-p_{-} \dot{x}^{-}\left(p_{-}\right)$.

Eq. (25) determines $\dot{x}^{-}\left(p_{-}\right)$; it is convenient before solving to take the limit $m \rightarrow 0$, where it reduces to $G_{\mu \nu} \dot{x}^{\mu} \dot{x}^{\nu}=0$. Then

$\dot{x}^{-}=\frac{\sqrt{1-h_{--} v^{2}}-1}{h_{--}}$.
In the $m \rightarrow 0$ limit at fixed $p_{-}$the effective Lagrangian becomes

$$
\begin{aligned}
& \mathcal{L}^{\prime} \rightarrow-p_{-} \dot{x}^{-} \\
& =p_{-}\left\{\frac{v^{2}}{2}+\frac{h_{--} v^{4}}{8}+\frac{h_{--}^{2} v^{6}}{16}+\ldots\right\} \\
& =\frac{N_{2}}{2 R} v^{2}+\frac{15}{16} \frac{N_{1} N_{2}}{R^{3} M^{9}} \frac{v^{4}}{r^{7}}+\frac{225}{64} \frac{N_{1}^{2} N_{2}}{R^{5} M^{18}} \frac{v^{6}}{r^{14}}+\ldots
\end{aligned}
$$

What do we see from this expression 2 ?

1. The $v$ and $r$ dependences exactly match with the diagonal terms of (20) and the $N$ dependence agrees with the leading large- $N$ behavior $N^{L+1}$, where $L$ is the number of loops.

2. We see again, that the $v^{4} / r^{7}$ term agrees with the one-loop M(atrix)-theory result (15).

3. The absence of a two-loop term $g v^{4} / r^{10}$ is in agreement with our previous $\mathrm{M}$ (atrix)theory result.

4. There appears a new term with a coefficient 225/64 that should correspond to a two-loop term in $\mathrm{M}$ (atrix)-theory.

Now comes our second two-loop test to M(atrix)theory. We will ask M(atrix)-theory: can you reproduce the two-loop term with the $225 / 64$ coefficient for us? Carrying out the calculation of the $v^{6} / r^{14}$-term in $\mathrm{M}$ (atrix)-theory by extending the calculation of [1], we indeed find precisely the correct numerical coefficient. The details about the different contributions can be found in [2]. Next we have to reconstruct the $N$-dependence of this result. Remember, we were considering the scattering of two D0-branes in M(atrix)-theory. To get the right $N_{1}$ and $N_{2}$ dependence, we must consider the scattering of a group of $N_{1}$ D0-branes against $N_{2}$ D0-branes. We can easily reconstruct the $N$-dependence of this scattering process. In double line notation every graph involves three index loops and so is of order $N^{3}$. Terms proportional to $N_{1}^{3}$ or $N_{2}^{3}$ would only involve one block (graviton) and so could not depend on $r$.

${ }^{2}$ The conventions in this section are appropriate for hermitian $X$. 
Symmetry under the interchange of $N_{1}$ and $N_{2}$ determines that the $S U(2)$ result is multiplied by

$\frac{N_{1} N_{2}^{2}+N_{1}^{2} N_{2}}{2}$,

which agrees with the supergravity result for the term of interest. Finally, restoring the dependence on $M$ and $R$ the two-loop result of $\mathrm{M}$ (atrix)-theory is precisely the result found in the supergravity calculation (28)!.

We think that the agreement of both effective actions is fascinating since the results for the different contributions to the two-loop effective action of M(atrix)-theory are rather complicated but when added up they conspire in such a way that the M-theory result is exactly reproduced. We are having a hard time believing that this agreement is a result of supersymmetry and independent of the M(atrix)-model conjecture. Therefore, we would like to speculate that there is a different structure behind all this: eleven-dimensional Lorentz-invariance.

\section{Conclusions and Outlook}

M(atrix)-theory has passed several rather strong two-loop tests. First, we have shown that the $v^{4} / r^{10}$-term at two-loops vanishes as it has to be for the agreement with M-theory to be correct. In our second test we have compared the $v^{6} / r^{14}$-term with the M-theory result. This term in the effective action appearing at two-loops is non-vanishing and exactly agrees in both theories. This might be indicating that with $\mathrm{M}$ (atrix)theory we are on the right track.

Some of the many questions that will have to be answered in a near future are the following. In this lecture I have discussed M(atrix)-theory in flat space. Comparisons of M(atrix)-theory with supergravity in curved space have been discussed by several authors, see for example 18 20]. We think it will be important to find the right formulation of $\mathrm{M}$ (atrix)-theory in curved space. Finally, I would like to remark that for the two-loop calculations considered so far, the momentum transfer in the eleven-direction has been set equal to zero. It will be interesting to understand scattering processes of D0-branes with eleven-dimensional momentum transfer and to make contact with the work considered in [21].

It is a pleasure to acknowledge the collaboration of Melanie Becker, Joe Polchinski and Arkady Tseytlin on the work presented in this lecture. I would like to thank the organizers of the Strings ' 97 conference for the invitation to present these results. This research was supported by NSF grant PHY89-04035.

\section{REFERENCES}

1. K. Becker and M. Becker, A Two Loop Test of M(atrix) Theory, ITP preprint NSF-ITP97-047, hep-th/9705091, to appear in Nucl. Phys. B.

2. K. Becker, M. Becker, J. Polchinski and A. Tseytlin, Higher Order Scattering in $M(a-$ trix) Theory, preprint NSF-ITP-97-061, hepth/9706072.

3. C. M. Hull and P. K. Townsend, Unity of Superstring Dualities, Nucl. Phys. B438 (1995) 109, hep-th/9410167; E.Witten, String Theory Dynamics in Various Dimensions, Nucl. Phys. B443 (1995) 85, hep-th/9503124; J.H.Schwarz, The Power of M-Theory, Phys. Lett. 367 (1996) 97, hep-th/9510086.

4. T. Banks, W. Fischler, S. H. Shenker and L. Susskind, $M$ Theory as a Matrix Model: A Conjecture, Phys. Rev. D55 (1997) 5112, hep-th/9610043.

5. J. Polchinski, Dirichlet Branes and RamondRamond Charges, Phys. Rev. Lett. 75 (1995) 4724, hep-th/9510017.

6. M. Claudson and M. B. Halpern, Supersymmetric Ground State Wave Functions, Nucl. Phys. B250 (1985) 689; M. Baake, P. Reinicke and V. Rittenberg, Fierz Identities for Real Clifford Algebras and the Number of Supercharges J. Math. Phys. 26 (1985) 1070; R. Flume, On Quantum Mechanics with Extended Supersymmetry and Nonabelian Gauge Constraints, Ann. Phys. 164 (1985) 189.

7. B. de Wit, J. Hoppe and H. Nicolai, On the Quantum Mechanics of Supermembranes, Nucl. Phys. B305 (1988) 545.

8. P. K. Townsend, D-Branes from M-Branes, Phys. Lett. 373 (1996) 68, hep-th/9512062. 
9. D. Kabat and P.Pouliot, A Comment on Zero-Brane Quantum Mechanics, Phys. Lett. 77 (1996) 1004, hep-th 9603127; U. H. Danielsson, G. Ferretti and B. Sundborg, $D$ Particle Dynamics and Bound States, Int. J. Mod. Phys. A11 (1996) 5463, hepth/9603081.

10. L. Susskind, Another Conjecture about M(atrix) Theory, Stanford preprint SU-ITP-9711, hep-th/9704080.

11. L. Susskind's talk at this conference.

12. R. Dijkgraaf, E. Verlinde and H. Verlinde, Matrix String Theory, hepth/9703030.

13. M. R. Douglas, D. Kabat, P. Pouliot and S. H. Shenker, D-branes and Short Distances in String Theory, Nucl. Phys. B485 (1997) 85, hep-th/9608024.

14. G. Lifschytz and S. M. Mathur, Supersymmetry and Membrane Interactions in M(atrix) theory, hep-th/9612087.

15. L. F. Abbott, Introduction to the Background Field Method, Acta Phys. Polon. B13 (1982) 33; The Background Field Method Beyond One Loop, Nucl. Phys. 185 (1981) 189.

16. D. Berenstein and R. Corrado, M(atrix) Theory in Various Dimensions, Texas preprint UTTG-04-97, hep-th/9702108.

17. P. C. Aichelburg and R. U. Sexl, On the Gravitational Field of a Massless Particle, Gen. Rel. Grav. 2, No.4 (1971) 303.

18. M. R. Douglas, H. Ooguri and S. H. Shenker, Issues in M(atrix) Model Compactification, hep-th/9702203.

19. O. J. Ganor, R. Gopakumar and S. Ramgoolam, Higher Loop Effects in M(atrix) Orbifolds, Princeton preprint PUPT-1680, hepth/9705188.

20. M. R. Douglas, D-Branes and Matrix Theory in Curved Space, Lecture given at Strings '97, hep-th/9707228.

21. J.Polchinski and P.Pouliot, Membrane Scattering with M-Momentum Transfer, ITP preprint NSF-ITP-97-27, hep-th/9704029;

N. Dorey, V.V. Khoze, and M.P. Mattis, Multi-Instantons, Three-Dimensional Gauge Theory, and the Gauss-Bonnet-Chern Theorem, preprint NI-97020, hep-th/9704197;

T. Banks, W. Fischler, N. Seiberg, and L.
Susskind, Instantons, Scale Invariance, and Lorentz Invariance in Matrix Theory, Texas preprint UTTG-18-97, hep-th/9705190.

22. J. Zinn-Justin, "Quantum Field Theory and Critical Phenomena", Oxford Science Publications. 